\title{
Endothelial repair capacity and apoptosis are inversely related in obstructive sleep apnea
}

\author{
This article was published in the following Dove Press journal: \\ Vascular Health and Risk Management \\ 30 October 2009 \\ Number of times this article has been viewed
}

\author{
Sanja Jelic' \\ David J Lederer' \\ Tessa Adams' \\ Margherita Padeletti' \\ Paolo C Colombo ${ }^{2}$ \\ Phillip Factor ${ }^{1}$ \\ Thierry H Le Jemtel ${ }^{3}$ \\ 'Division of Pulmonary, Allergy, and \\ Critical Care Medicine, ${ }^{2}$ Division \\ of Cardiology, Columbia University \\ College of Physicians and Surgeons, \\ New York, NY, USA; ${ }^{3}$ Division of \\ Cardiology, Tulane University School \\ of Medicine, New Orleans, LA, USA
}

Correspondence: Sanja Jelic Columbia University College of Physicians and Surgeons, Division of Pulmonary, Allergy, and Critical Care Medicine, PH8 Center, Room I0I, 630 West I68th Street, New York, NY 10032, USA

Tel + I 212305759 I

Fax + I 2I2305 7072

Email sj366@columbia.edu
Purpose: To investigate the impact of obstructive sleep apnea (OSA) on endothelial repair capacity and apoptosis in the absence of potentially confounding factors including obesity.

Patients and methods: Sixteen patients with a body mass index $<30$ and newly diagnosed OSA and 16 controls were studied. Circulating levels of endothelial progenitor cells, a marker of endothelial repair capacity, and endothelial microparticles, a marker of endothelial apoptosis, were quantified before and after four-week therapy with continuous positive airway pressure (CPAP). Endothelial cell apoptotic rate was also quantified in freshly harvested venous endothelial cells. Vascular reactivity was measured by flow-mediated dilation.

Results: Before treatment, endothelial microparticle levels were greater and endothelial progenitor cell levels were lower in patients with OSA than in controls $(P<0.001$ for both). Levels of endothelial microparticles and progenitors cells were inversely related $(\mathrm{r}=-0.67, P<0.001)$. Endothelial progenitor cell levels increased after effective treatment $(P=0.036)$.

Conclusions: In the absence of any co-morbid conditions including obesity, OSA alone impairs endothelial repair capacity and promotes endothelial apoptosis. These early endothelial alterations may underlie accelerated atherosclerosis and increased cardiovascular risk in OSA.

Keywords: sleep apnea, endothelium, apoptosis, endothelial repair capacity

\section{Introduction}

Accelerated atherosclerosis and cardiovascular morbidity may result from continuous damage to the vascular endothelium in patients with obstructive sleep apnea (OSA). ${ }^{1-8}$ Patients with coronary artery disease (CAD) exhibit increased rates of endothelial apoptosis and reduced endothelial repair capacity that contribute to continuous endothelial damage. ${ }^{9,10}$ Whether OSA independently affects endothelial apoptosis and repair capacity remains controversial. ${ }^{11-17}$ Increased rate of endothelial apoptosis has been reported in patients with OSA by some investigators while others have reported similar levels of apoptotic microparticles in patients with OSA and controls. ${ }^{11,12,15-17}$ The data regarding endothelial repair capacity in patients with OSA are also controversial: Endothelial repair capacity was reported to be intact by some investigators and reduced by others. ${ }^{8,13,14}$ Of note the presence of co-morbid conditions which are frequently associated with OSA and known to affect the vascular endothelium was not systematically excluded in all above mentioned studies.

Accordingly, the present study was undertaken to determine the effects of OSA on endothelial apoptosis and repair capacity in the absence of any conditions known to affect the vascular endothelium. In particular, we exclusively studied nonobese patients 
with OSA as obesity alone affects the vascular endothelium. ${ }^{18}$ We hypothesized that the rate of endothelial apoptosis is greater and repair capacity lower in patients with OSA than in healthy controls matched for age, gender and body mass index (BMI), and that continuous positive airway pressure (CPAP) therapy decreases the rate of endothelial apoptosis while improving endothelial repair capacity. The rate of endothelial apoptosis was quantified by measuring: a) levels of circulating endothelial microparticles (EMP) that are shed directly from apoptotic endothelial cells, and b) the rate of apoptotic nuclei in freshly harvested venous endothelial cells. Endothelial repair capacity was assessed by quantifying solely immature endothelial cells. ${ }^{19-25}$ Lastly, flow-mediated dilation was assessed as the functional correlate of endothelial apoptosis and repair capacity in OSA. Measurements were repeated after CPAP therapy for four weeks.

\section{Methods}

\section{Study population}

Patients who were evaluated in the Sleep Disorders Center at the New York Presbyterian Hospital for evaluation of sleep-disordered breathing between March 2006 and April 2009 were prospectively screened for the study. Obesity was defined as $\mathrm{BMI} \geq 30$. Patients with newly diagnosed OSA defined as an apnea-hypopnea index (AHI) of five or more obstructive events per hour of sleep and BMI < 30 who were free of conditions known to affect the vascular endothelium were eligible for the study. Patients with dyslipidemias, diabetes mellitus, cardiovascular, neurological, pulmonary, and renal diseases were ineligible for the study. Former and current smokers and patients receiving medications or nutritional supplements were also ineligible.

Controls were nonsmoking healthy subjects who were not receiving medications or nutritional supplements. Control subjects were recruited from the community through advertising and were matched to patients for gender, age (within four years), and BMI (within 15\%). Six patients with OSA in the present study were included in the previous study conducted in our Sleep Disorders Center. ${ }^{8}$ The Columbia University Committee on Human Research approved the study. All study participants signed a written informed consent.

\section{Study protocol}

Nocturnal polysomnography was performed in all study participants as previously described. ${ }^{8} \mathrm{AHI}$ was defined as the number of obstructive apnea plus hypopnea episodes per hour of sleep. Endothelial cells harvesting, blood sample collection, and flow-mediated dilation (FMD) were performed between
9:00 and 11:00 AM within 48 hours of polysomnography while study participants were in a fasting state. All experimental procedures were repeated after a four-week treatment period in all OSA patients. Adherence with CPAP was defined as CPAP use $\geq 4$ hours daily. ${ }^{1}$ Adherence was assessed by using CPAP device with compliance software.

\section{Harvesting and immunofluorescence for endothelial cell apoptosis}

A 20-gauge angiocath was inserted into a forearm vein. Under sterile conditions, three J-shaped vascular guide wires (Arrow, Reading, PA) were sequentially advanced into the vein up to $10 \mathrm{~cm}$. Tips of the wires were removed and washed in endothelial cell dissociation buffer kept at $4{ }^{\circ} \mathrm{C}$. Endothelial cells were recovered by centrifugation and fixed with $3.7 \%$ formaldehyde in phosphate-buffered saline (PBS) for $10 \mathrm{~min}$, washed twice with PBS, transferred to poly-L-lysine coated slides (Sigma, St. Louis, MO), and air-dried at $37^{\circ} \mathrm{C}$. The slides were stored at $-80{ }^{\circ} \mathrm{C}$ until analyzed. Apoptotic cell nuclei were identified using in situ apoptosis peroxidase detection kit, the terminal deoxynucleotide transferase-mediated dUTP nick-end labeling (TUNEL). (Details regarding immunofluorescence can be found in the Data Supplement).

\section{Flow cytometry for circulating endothelial progenitor cells and apoptotic microparticles}

EPC and EMP were quantified in venous blood by flow cytometry. EPC were defined as cells positive for monoclonal antibodies against human KDR (kinase insert domain receptor), CD34, and CD133 (Figures 1A and 1B in the Data Supplement). ${ }^{19,22-25}$ EMP were defined as particles $\leq 1.5 \mu \mathrm{m}$ in size positive for monoclonal antibodies against $\mathrm{CD} 31$ and negative for CD42b (Figure 2A and 2B in the Data Supplement). ${ }^{26}$ (Details regarding flow cytometry can be found in the Data Supplement).

\section{Brachial artery flow-mediated dilation}

Vascular reactivity of the brachial artery was assessed in the contra-lateral arm to the endothelial harvesting site by FMD according to the guidelines of the International Brachial Artery Reactivity Task Force. ${ }^{27}$ (Details regarding FMD can be found in the Data Supplement).

\section{Statistical analysis}

Continuous data are presented as mean \pm standard deviation or median (interquartile range). Categorical data are 
presented as percentage. To determine whether endothelial apoptosis and repair capacity differ between patients with OSA and controls, EMP and EPC levels were compared between groups using Wilcoxon rank sum tests and Fisher exact tests. To determine whether CPAP therapy affects EMP and EPC, we compared these measures before and after CPAP therapy using Wilcoxon signed rank tests. Statistical significance was assumed when null hypothesis could be rejected at $P<0.05$. Statistical analysis was performed with the use of SAS software (version 9.1; SAS Institute, Cary, NC).

\section{Results}

Sixteen patients with OSA and 16 control subjects were studied. The clinical and laboratory characteristics of study participants are summarized in Table 1. Patients and control subjects were similar regarding age, gender, BMI, systemic blood pressure, fasting blood glucose and total cholesterol levels. OSA patients had significantly lower arterial oxyhemoglobin saturation $\left(\mathrm{SaO}_{2}\right)$ nadir during sleep and had more daytime sleepiness as measured by Epworth Sleepiness Scale than control subjects. Control subjects had an AHI $<5$ and spent no time during sleep with $\mathrm{SaO}_{2}<90 \%$.

\section{Endothelial apoptosis and repair capacity at baseline}

Before treatment, levels of circulating EPC, a marker of endothelial repair capacity, were significantly lower and levels of circulating EMP, a marker of endothelial apoptosis, were significantly greater in patients with OSA than in controls
(Figures 1A and 1B). Apoptotic nuclei in harvested venous endothelial cells were not detectable in all specimens obtained from patients with OSA and controls. Levels of circulating EMP and EPC were inversely related in the entire cohort (Figure 2).

\section{Effects of CPAP therapy on endothelial apoptosis and repair capacity}

Eight patients used CPAP for four weeks and eight patients declined CPAP therapy due to discomfort. The average daily use of CPAP was $5.8 \pm 2.1$ hours (range 2-8 hours). Seven patients used CPAP $\geq 4$ hours daily for an average of $6.4 \pm 1.5$ hours. The one remaining patient used CPAP for an average of two hours daily. Age, BMI, $\mathrm{AHI}, \mathrm{SaO}_{2}$ nadir, $\mathrm{t}<\mathrm{SaO}_{2} 90 \%$, daytime sleepiness, blood pressure, fasting glucose and total cholesterol levels as well as baseline EMP and EPC levels were similar in patients who accepted CPAP and patients who declined CPAP. Body weight and systolic and diastolic blood pressure remained unchanged during CPAP therapy.

CPAP therapy significantly increased EPC levels and tended to decrease EMP levels in patients with OSA compared with their baseline values (Figures $1 \mathrm{C}$ and 1D). After effective treatment (CPAP $\geq 4$ hours daily), EPC levels were similar in patients with OSA and controls $(P=0.92)$. Levels of EMP and EPC were unchanged in patients who declined CPAP and in one patient who used CPAP $<4$ hours daily. Apoptotic nuclei in harvested venous endothelial cells remained undetectable in all specimens obtained from adherent and nonadherent OSA patients.

Table I Baseline characteristics of patients with obstructive sleep apnea and control subjects

\begin{tabular}{|c|c|c|c|}
\hline & $\begin{array}{l}\text { OSA patients } \\
(n=16)\end{array}$ & $\begin{array}{l}\text { Control subjects } \\
(n=I 6)\end{array}$ & $P$ value \\
\hline Age (years) & $36 \pm 9$ & $36 \pm 11$ & NS \\
\hline Gender (\% female) & 44 & 50 & NS \\
\hline Body mass index $\left(\mathrm{kg} / \mathrm{m}^{2}\right)$ & $27 \pm 2$ & $26 \pm 3$ & NS \\
\hline Apnea-Hypopnea Index (events/h of sleep) & $22 \pm 24$ & $0.1 \pm 0.5$ & $<0.001$ \\
\hline $\mathrm{SaO}_{2} \operatorname{nadir}(\%)$ & $88 \pm 5$ & $97 \pm 1$ & $<0.001$ \\
\hline $\mathrm{t}<\mathrm{SaO}_{2} 90 \%(\% \text { of the total sleep time })^{\mathrm{a}}$ & $1.4 \pm 2.6$ & $0 \pm 0$ & $<0.001$ \\
\hline Epworth Sleepiness Scale Score & $12 \pm 5$ & $4 \pm 3$ & $<0.001$ \\
\hline Systolic blood pressure $(\mathrm{mm} \mathrm{Hg})$ & $118 \pm 13$ & $120 \pm 10$ & NS \\
\hline Diastolic blood pressure (mm Hg) & $71 \pm 10$ & $73 \pm 10$ & NS \\
\hline Fasting glucose (mg/dL) & $90 \pm 16$ & $87 \pm 10$ & NS \\
\hline Total cholesterol (mg/dL) & $183 \pm 56$ & $188 \pm 45$ & NS \\
\hline
\end{tabular}

Notes: Data are presented as mean \pm standard deviation or $\%$. ${ }^{a}$ Time spent below $\mathrm{SaO}_{2}$ of $90 \%$ during sleep. Abbreviations: NS, not significant; OSA, obstructive sleep apnea; $\mathrm{SaO}_{2}$, arterial oxyhemoglobin saturation. 
A

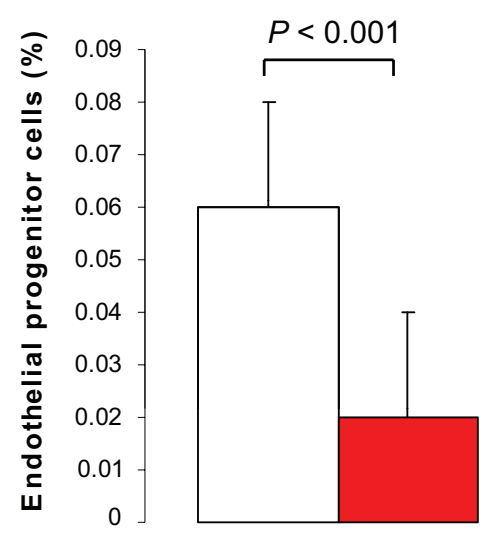

B

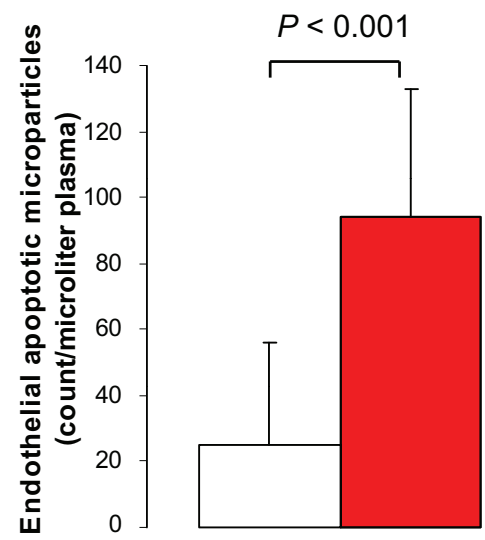

C

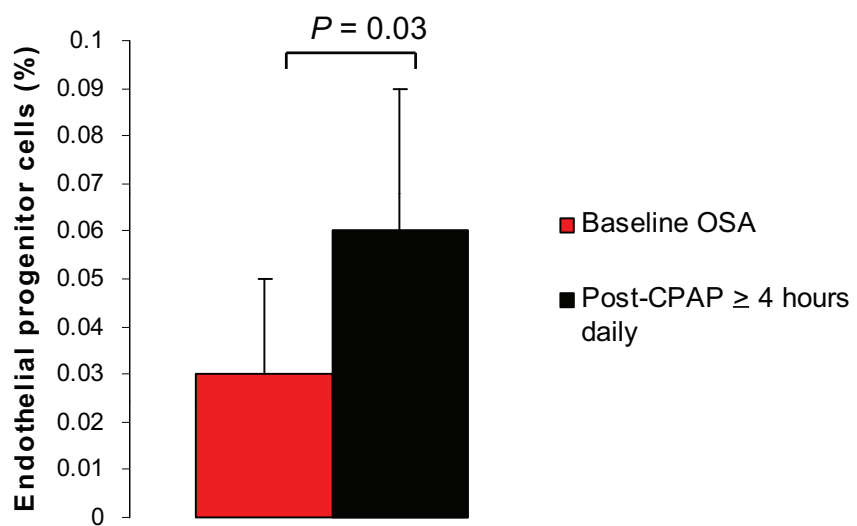

D

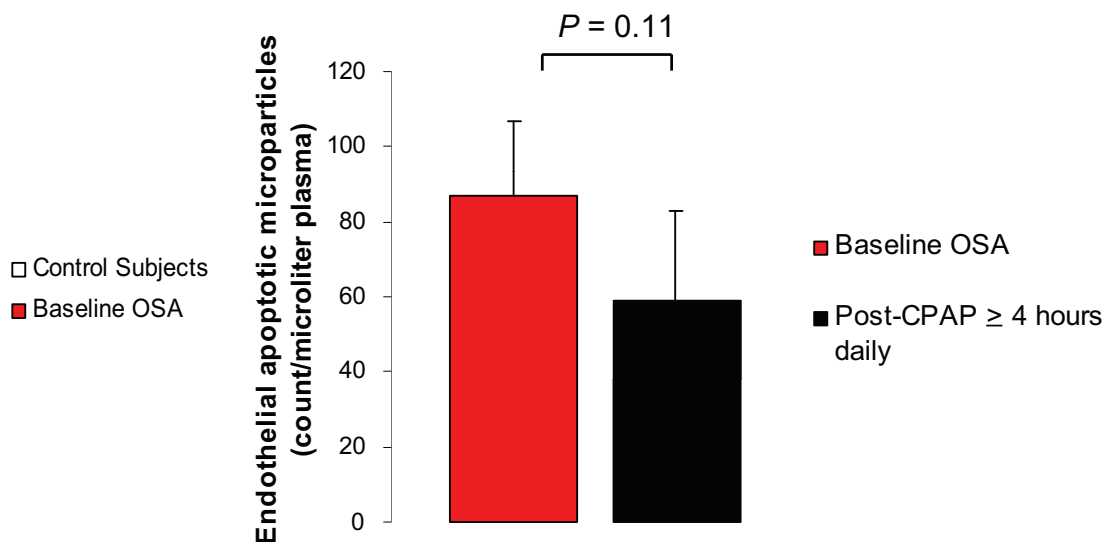

Figure I Levels of circulating endothelial progenitor cells (EPC) and apoptotic microparticles (EMP) in healthy controls and patients with obstructive sleep apnea (OSA) before and after treatment with continuous positive airway pressure (CPAP). Baseline levels of EPC were lower in OSA patients $(n=16)$ than in controls $(n=16)$ A) while levels of EMP were greater B). Levels of EPC increased significantly C) while levels of EMP tended to decreased $D$ ) when patients adhered with CPAP $\geq 4$ hours daily ( $=7$ ).

\section{Markers of endothelial apoptosis and} repair capacity and flow-mediated dilation Baseline brachial artery FMD, an indirect marker of endothelial nitric oxide-mediated reactivity, was lower in patients with OSA than in controls: $3.6 \pm 2.6$ vs $9.1 \% \pm 4.5 \%,(P=0.004)$. Effective treatment (CPAP $\geq 4$ hours daily) tended to increase FMD $(3.3 \pm 2.1$ vs $7.4 \% \pm 3.9 \%$, $P=0.06)$. In the absence of effective CPAP treatment, FMD remained unchanged. No differences in the resting brachial diameter and percent reactive hyperemia after cuff deflation were observed between patients with OSA and controls. Baseline circulating EMP levels were inversely related to FMD (Spearman correlation coefficient adjusted for $\mathrm{BMI}=-0.43, P=0.02$ ) (Figure 3 ). Baseline circulating EPC levels did not correlate with FMD ( $\mathrm{r}=0.30, P=0.13)$.

\section{Discussion}

The present data indicate that OSA independently promotes vascular endothelial apoptosis and impairs endothelial repair capacity. Effective CPAP therapy improves endothelial repair capacity. Enhanced endothelial apoptosis and impaired repair capacity may hasten the progression of atherosclerosis and thereby the development of hypertension, ischemic stroke or myocardial ischemia.

\section{Endothelial apoptosis in OSA}

Elevated circulating EMP levels in otherwise healthy patients with OSA when compared to controls are strong evidence for enhanced endothelial apoptosis in OSA. The significant correlation between circulating EMP levels and endothelium-dependent vasodilation suggests that enhanced endothelial apoptosis results in endothelial damage thereby 


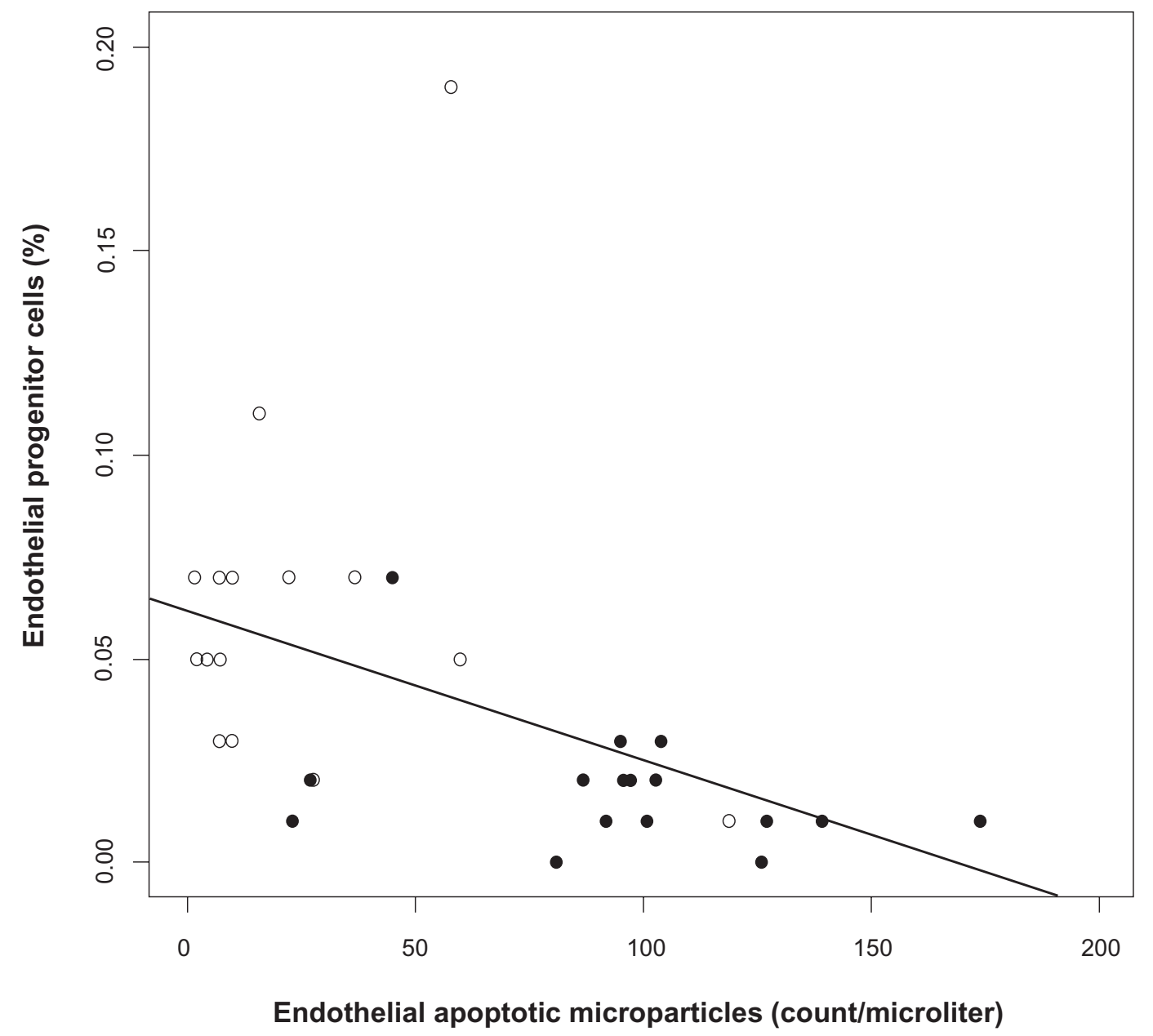

Figure 2 Relation between endothelial apoptotic rate and endothelial repair capacity in obstructive sleep apnea (OSA). Levels of circulating endothelial progenitor cells (EPC) and microparticles (EMP) are inversely related at baseline (Spearman correlation coefficient $r=-0.67, P<0.00 \mathrm{I}$ ). OSA patients (closed circles), healthy controls (open circles).

depressing vascular endothelial function. The small number of venous endothelial cells that could be studied for apoptosis (100-150) most likely accounts for our inability to demonstrate apoptosis in harvested endothelial cells by TUNEL assay. Direct demonstration of apoptosis will require harvesting considerably more endothelial cells than we did in view of the relatively low endothelial apoptotic rate. ${ }^{28}$

Endothelial cell-derived apoptotic microparticles are directly shed from mature endothelial cells during apoptosis. Levels of circulating EMP inversely correlate with endothelium-dependent dilation in patients with CAD. ${ }^{9}$ Elevated levels of circulating EMP have been reported in obese women who were not systematically evaluated for OSA. ${ }^{29}$ Considering a high prevalence of OSA in obesity, unrecognized OSA may have contributed to increased rate of endothelial apoptosis. ${ }^{29}$ Repetitive episodes of hypoxia/ reoxygenation, such as observed with transient cessation of breathing in OSA, may promote endothelial apoptosis by activating cell death receptors and mitochondria-dependent apoptotic pathways. ${ }^{30,31}$ Elevated circulating EMP levels also directly affect the vascular endothelium. ${ }^{32,33}$ Exposure of rat aortic rings to increasing concentrations of isolated EMP derived from cultured human endothelial cells or patients with myocardial ischemia alters endothelium-dependent vasodilation and nitric oxide production while simultaneously increasing superoxide production. ${ }^{32,34}$ Increased concentrations of EMP also impair angiogenesis in vitro. ${ }^{32}$ When isolated from atherosclerotic plaques during carotid endarterectomy, EMP are highly thrombogenic and promote plaque formation and propagation. ${ }^{35}$

Elevated levels of circulating EMP in nonobese patients with OSA who are free of overt cardiovascular diseases strongly suggest that OSA alone promotes endothelial apoptosis. Increased levels of circulating EMP may underlie altered endothelial function and coagulation homeostasis in OSA. 


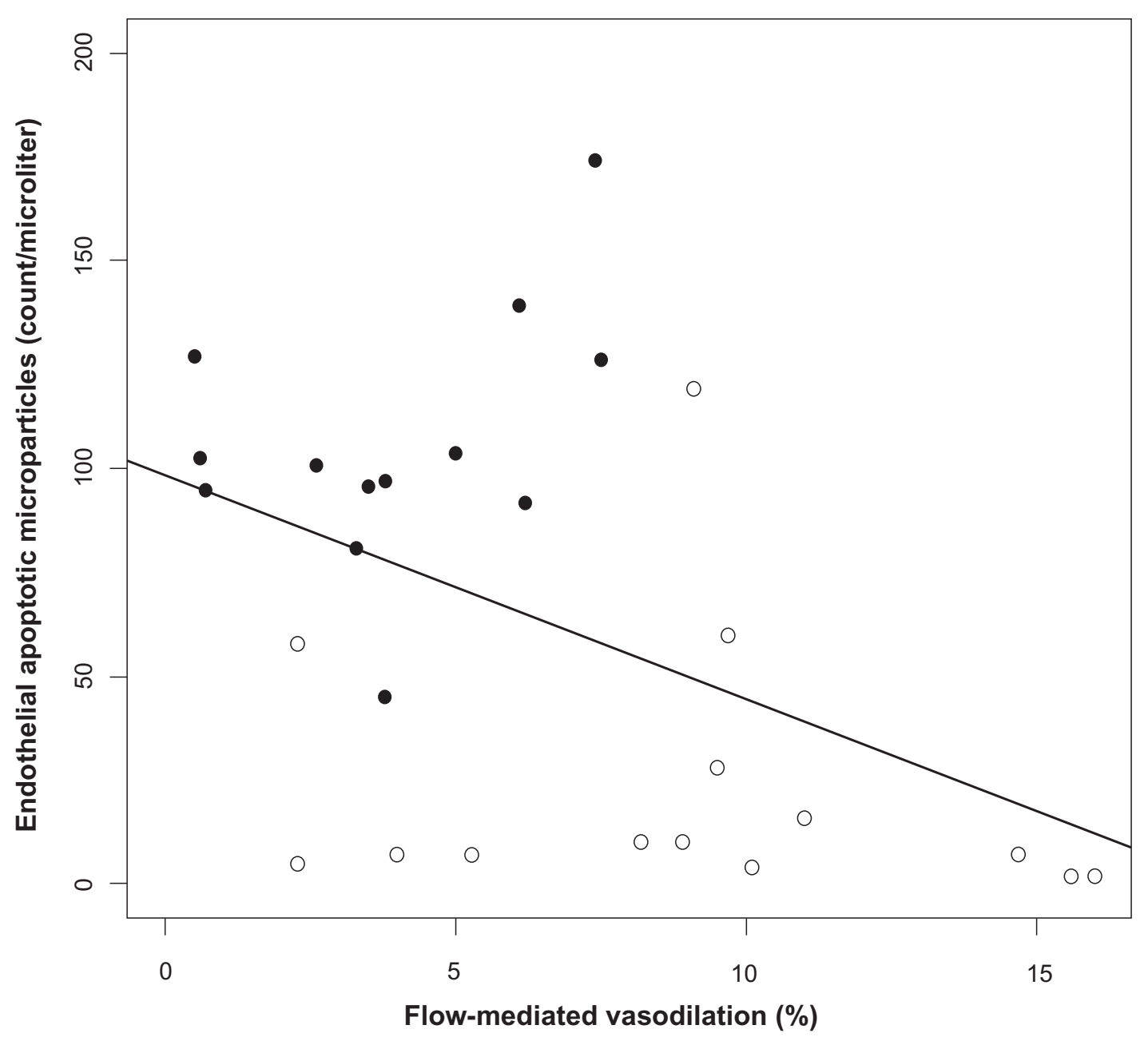

Figure 3 Relation between endothelial apoptotic rate and flow-mediated dilation in obstructive sleep apnea (OSA). Levels of circulating apoptotic microparticles correlate inversely with flow-mediated dilation in OSA (Spearman correlation coefficient adjusted for body mass index $r=-0.43, P=0.02$ ). OSA patients (closed circles), healthy controls (open circles).

\section{Endothelial repair capacity in OSA}

Intact endothelial repair capacity may offset the progressive loss of endothelial cells due to repetitive hypoxia/reoxygenation in patients with OSA. Expression of CD34, CD133 and KDR is currently required for EPC identification by most investigators. ${ }^{19,22}$ A recent controversial report has questioned whether $\mathrm{CD} 34^{+}, \mathrm{CD} 133^{+}$, and VEGFR $2^{+}$cells are endothelial or hematopoietic progenitors. ${ }^{36}$ Independently from their controversial capacity to form vessel-like structures in vitro, $\mathrm{CD} 34^{+}, \mathrm{CD}_{133^{+}}$, and VGEFR $2^{+}$undoubtedly restore endothelial lining in vivo. ${ }^{37-40} \mathrm{~A}$ decreased level of such cells in OSA is consistent with reduced endothelial repair capacity that can be reversed by CPAP therapy.

Reduced EPC levels compromise endothelial repair by reducing availability of angiogenic growth factors and the pool of endothelial cells with proliferative capacity. ${ }^{22}$ Reduced EPC level and activity are associated with impaired endothelial function and increased risk for vascular events in patients with CAD. ${ }^{10,41}$ Mobilization of EPC from the bone marrow entails adequate NO production that is reduced in patients with OSA..$^{6-84}$ Reduced circulating EPC levels limit further NO production since EPC are an important source of NO production at the site of ischemia/reperfusion induced endothelial injury. ${ }^{43}$ The inverse correlation between circulating levels of EMP and EPC supports the view that enhanced endothelial apoptosis may exhaust the repair capacity of the vascular endothelium in OSA thereby resulting in continuous endothelial damage.

\section{The effect of treatment for OSA on endothelial apoptosis and repair capacity} Adherence with CPAP therapy $\geq 4$ hours nightly for four weeks exerted beneficial effects on endothelial repair capacity but did not significantly affect endothelial apoptosis or 
FMD. Endothelial apoptosis appears to be a rare event in both patients with OSA and controls. Small number of OSA patients who adhered with CPAP therapy may have contributed to the lack of significant difference between baseline and post-CPAP measurements of endothelial apoptosis and FMD. The beneficial effects of CPAP therapy on endothelial repair capacity and apoptosis need to be confirmed in a larger study than the present one that will include multiple centers and randomized allocation of CPAP therapy.

\section{Study limitations}

The study population was small due to the difficulty in recruiting nonobese OSA patients who are free of any condition known to affect vascular endothelium. Over $70 \%$ of patients diagnosed with OSA in our Sleep Disorders Center have a $\mathrm{BMI} \geq 30$ and the majority have clinical evidence of cardiovascular disease. We did not study the capacity of EPC from OSA patients to form colonies in vitro. Reduced circulating EPC levels have been associated with ongoing endothelial damage and reduced function of the EPC in vitro. ${ }^{10,44-46}$

\section{Conclusions}

In summary, OSA independently promotes endothelial apoptosis and impairs endothelial repair capacity. Enhanced endothelial apoptosis and impaired repair capacity may contribute to the high prevalence of vascular conditions in patients with OSA.

\section{Disclosures}

The authors were supported by grants from the Irving Center for Clinical Research RR-0645, and American Lung Association CU-52259701 (SJ). The authors report no conflicts of interest in this work.

\section{References}

1. Marin JM, Carrizo SJ, Vicente E, Agusti AG. Long-term cardiovascular outcomes in men with obstructive sleep apnoea-hypopnoea with or without treatment with continuous positive airway pressure: an observational study. Lancet. 2005;365:1046-1053.

2. Drager LF, Bortolotto LA, Lorenzi MC, Figueiredo AC, Krieger EM, Lorenzi-Filho G. Early signs of atherosclerosis in obstructive sleep apnea. Am J Respir Crit Care Med. 2005;172:613-618.

3. Drager LF, Bortolotto LA, Figueiredo AC, Krieger EM, Lorenzi GF. Effects of continuous positive airway pressure on early signs of atherosclerosis in obstructive sleep apnea. Am J Respir Crit Care Med. 2007;176:706-712.

4. Minoguchi K, Yokoe T, Tazaki T, et al. Increased carotid intima-media thickness and serum inflammatory markers in obstructive sleep apnea. Am J Respir Crit Care Med. 2005;172:625-630.

5. Savransky V, Nanayakkara A, Li J, et al. Chronic intermittent hypoxia induces atherosclerosis. Am J Respir Crit Care Med. 2007;175: 1290-1297.
6. Kato M, Roberts-Thomson P, Phillips BG, et al. Impairment of endothelium-dependent vasodilation of resistance vessels in patients with obstructive sleep apnea. Circulation. 2000;102:2607-2610.

7. Ip MS, Tse HF, Lam B, Tsang KW, Lam WK. Endothelial function in obstructive sleep apnea and response to treatment. Am J Respir Crit Care Med. 2004;169:348-353.

8. Jelic S, Padeletti M, Kawut SM, et al. Inflammation, oxidative stress and repair capacity of the vascular endothelium in obstructive sleep apnea. Circulation. 2008;117:2270-2278.

9. Werner N, Wassmann S, Ahlers P, Kosiol S, Nickenig G. Circulating CD31+/annexin $\mathrm{V}+$ apoptotic microparticles correlate with coronary endothelial function in patients with coronary artery disease. Arterioscler Thromb Vasc Biol. 2006;26:112-116.

10. Werner N, Wassmann S, Ahlers P, et al. Endothelial progenitor cells correlate with endothelial function in patients with coronary artery disease. Basic Res Cardiol. 2007;102:565-571.

11. El Solh AA, Akinnusi ME, Baddoura FH, Mankowski CR. Endothelial cell apoptosis in obstructive sleep apnea: a link to endothelial dysfunction. Am J Respir Crit Care Med. 2007;175:1186-1191.

12. El Solh AA, Akinnusi ME, Berim IG, Peter AM, Paasch LL, Szarpa KR. Hemostatic implications of endothelial cell apoptosis in obstructive sleep apnea. Sleep Breath. 2008;12:331-337.

13. de la Peña M, Barceló A, Barbe F, et al. Endothelial function and circulating endothelial progenitor cells in patients with sleep apnea syndrome. Respiration. 2007;76:28-32.

14. Martin K, Stanchina M, Kouttab N, Harrington EO, Rounds S. Circulating endothelial cells and endothelial progenitor cells in obstructive sleep apnea. Lung. 2008;186:145-150.

15. Goon PK, Watson T, Lip GY. Circulating endothelial cells in obstructive sleep apnea: an important methodological lesson. Am J Respir Crit Care Med. 2007; 175:1346.

16. Tzouvelekis A, Kotsianidis I, Steiropoulos P, Bouros D. Role of CD146 in detection of apoptotic circulating endothelial cells in obstructive sleep apnea. Am J Respir Crit Care Med. 2007;175:1346-1347.

17. Ayers L, Ferry B, Craig S, Nicoll D, Stradling JR, Kohler M. Circulating cell-derived microparticles in patients with minimally symptomatic obstructive sleep apnoea. Eur Respir J. 2009;33:574-580.

18. Poirier P, Giles TD, Bray GA, et al; American Heart Association; Obesity Committee of the Council on Nutrition, Physical Activity, and Metabolism. Obesity and cardiovascular disease: pathophysiology, evaluation, and effect of weight loss: an update of the 1997 American Heart Association Scientific Statement on Obesity and Heart Disease from the Obesity Committee of the Council on Nutrition, Physical Activity, and Metabolism. Circulation. 2006;113:898-918.

19. Urbich C, Dimmeler S. Endothelial progenitor cells: characterization and role in vascular biology. Circ Res. 2004;95:343-353.

20. Del Papa N, Colombo G, Fracchiolla N, et al. Circulating endothelial cells as a marker of ongoing vascular disease in systemic sclerosis. Arthritis Rheum. 2004;50:1296-1304.

21. Timmermans F, Van Hauwermeiren F, De Smedt M, et al. Endothelial outgrowth cells are not derived from CD133+ cells or CD45+ hematopoietic precursors. Arterioscler Thromb Vasc Biol. 2007;27:1572-1579.

22. Zampetaki A, Kirton JP, Xu Q. Vascular repair by endothelial progenitor cells. Cardiovasc Res. 2008;78:413-421.

23. Asahara T, Murohara T, Sullivan A, et al. Isolation of putative progenitor endothelial cells for angiogenesis. Science. 1997;275: 964-967.

24. Werner N, Kosiol S, Schiegl T, et al. Circulating endothelial progenitor cells and cardiovascular outcomes. N Engl J Med. 2005;353: 999-1007.

25. Peichev M, Naiyer AJ, Pereira D, et al. Expression of VEGFR-2 and AC133 by circulating human CD34(+) cells identifies a population of functional endothelial precursors. Blood. 2000;95:952-958.

26. Ferreira AC, Peter AA, Mendez AJ, et al. Postprandial hypertriglyceridemia increases circulating levels of endothelial cell microparticles. Circulation. 2004;110:3599-3603. 
27. Corretti MC, Anderson TJ, Benjamin EJ, et al. International Brachial Artery Reactivity Task Force. Guidelines for the ultrasound assessment of endothelial-dependent flow-mediated vasodilation of the brachial artery: a report of the International Brachial Artery Reactivity Task Force. J Am Coll Cardiol. 2002;39:257-265.

28. Kalashnik L, Bridgeman CJ, King AR, et al. A cell kinetic analysis of human umbilical vein endothelial cells. Mech Ageing Dev. 2000;120: 23-32.

29. Esposito K, Ciotola M, Schisano B, et al. Endothelial microparticles correlate with endothelial dysfunction in obese women. J Clin Endocrinol Metab. 2006;91:3676-3679.

30. Zhang Y, Zhang X, Park TS, Gidday JM. Cerebral endothelial cell apoptosis after ischemia-reperfusion: role of PARP activation and AIF translocation. J Cereb Blood Flow Metab. 2005;25:868-877.

31. Dhar-Mascareno M, Cárcamo JM, Golde DW. Hypoxia-reoxygenationinduced mitochondrial damage and apoptosis in human endothelial cells are inhibited by vitamin C. Free Radic Biol Med. 2005;38: 1311-1322.

32. Brodsky SV, Zhang F, Nasjletti A, Goligorsky MS. Endothelium-derived microparticles impair endothelial function in vitro. Am J Physiol Heart Circ Physiol. 2004;286:H1910-H1915.

33. Mezentsev A, Merks RM, O'Riordan E, et al. Endothelial microparticles affect angiogenesis in vitro: role of oxidative stress. Am J Physiol Heart Circ Physiol. 2005;289:H1106-H1114.

34. Boulanger CM, Scoazec A, Ebrahimian T, et al. Circulating microparticles from patients with myocardial infarction cause endothelial dysfunction. Circulation. 2001;104:2649-2652.

35. Leroyer AS, Isobe $H$, Lesèche $G$, et al. Cellular origins and thrombogenic activity of microparticles isolated from human atherosclerotic plaques. J Am Coll Cardiol. 2007;49:772-777.

36. Case J, Mead LE, Bessler WK, et al. Human CD34+AC133+VEGFR-2+ cells are not endothelial progenitor cells but distinct, primitive hematopoietic progenitors. Exp Hematol. 2007;35:1109-1118.
37. Crosby JR, Kaminski WE, Schatteman G, et al. Endothelial cells of hematopoietic origin make a significant contribution to adult blood vessel formation. Circ Res. 2000;87:728-730.

38. Gothert JR, Gustin SE, van Eekelen JA, et al. Genetically tagging endothelial cells in vivo: bone marrow-derived cells do not contribute to tumor endothelium. Blood. 2004;104:1769-1777.

39. Peters BA, Diaz LA, Polyak K, et al. Contribution of bone marrowderived endothelial cells to human tumor vasculature. Nat Med. 2005;11:261-262.

40. Zentilin L, Tafuro S, Zacchigna S, et al. Bone marrow mononuclear cells are recruited to the sites of VEGF-induced neovascularization but are not incorporated into the newly formed vessels. Blood. 2006;107:3546-3554.

41. Hill JM, Zalos G, Halcox JP, et al. Circulating endothelial progenitor cells, vascular function, and cardiovascular risk. $N$ Engl J Med. 2003;348:593-600.

42. Aicher A, Heeschen C, Mildner-Rihm C, et al. Essential role of endothelial nitric oxide synthase for mobilization of stem and progenitor cells. Nat Med. 2003;9:1370-1376.

43. Ii M, Nishimura H, Iwakura A, et al. Endothelial progenitor cells are rapidly recruited to myocardium and mediate protective effect of ischemic preconditioning via "imported" nitric oxide synthase activity. Circulation. 2005;111:1114-1120.

44. Schmidt-Lucke C, Rössig L, Fichtlscherer S, et al. Reduced number of circulating endothelial progenitor cells predicts future cardiovascular events: proof of concept for the clinical importance of endogenous vascular repair. Circulation. 2005;111:2981-2987.

45. Grisar J, Aletaha D, Steiner CW, et al. Depletion of endothelial progenitor cells in the peripheral blood of patients with rheumatoid arthritis. Circulation. 2005;111:204-211.

46. Vasa M, Fichtlscherer S, Aicher A, et al. Number and migratory activity of circulating endothelial progenitor cells inversely correlate with risk factors for coronary artery disease. Circ Res. 2001;89:E1-E7. 


\section{Data supplement \\ Methods}

Immunofluorescence for endothelial cell apoptosis

Endothelial cells were permeabilized in PBS/0.5\% Triton X-100. Polyclonal anti-von Willebrand factor antibodies (DAKO, Glostrup, Denmark) were used, followed by Texas red-conjugated secondary antibodies to identify endothelial cells. Nuclei were stained with diaminophenylindole (DAPI) (Molecular Probes, Carlsbad, CA). Apoptotic cell nuclei were identified using in situ apoptosis peroxidase detection kit, the terminal deoxynucleotide transferase-mediated dUTP nick-end labeling (TUNEL) according to the manufacturer's instruction (Intergen). Nucleated endothelial cells were identified by red (von Willebrand factor staining) and blue fluorescence (DAPI DNA staining), and apoptosis was detected by green fluorescence (TUNEL). Apoptotic endothelial cells were detected by co-localized red and green fluorescence. Slides from study participants were stained concurrently with two slides of human umbilical venous endothelial cells (HUVEC) that served as positive and negative control. For negative controls, terminal deoxynucleotidyltransferase was omitted from the labeling mixture. For positive control, HUVEC slides were treated with DNase buffer for five minutes. Endothelial cells were analyzed with a fluorescent microscope under identical conditions (Nikon Eclipse E600, Melville, NY), and were captured by digital camera (Q Imaging Retiga EXi, Surrey, BC, Canada). The reader was blinded to subjects' identity. Slides were systematically read left to right and top to bottom. The number of TUNELpositive endothelial cells was scored in 10 randomly chosen high-power fields.

\section{Flow cytometry for circulating endothelial progenitor cells}

Twelve $\mathrm{mL}$ of venous blood was withdrawn from a forearm vein via the angiocath inserted for the endothelial harvesting. Mononuclear cells were isolated by densitygradient centrifugation with Ficoll (Sigma) and counted using a Coulter Counter (Beckman Coulter, Fullerton, CA) within one hour of blood sample collection. One million mononuclear cells were aliquoted and incubated with $15 \mu \mathrm{L}$ mouse serum (Sigma) at room temperature to block nonspecific binding of antibodies. Mononuclear cells were incubated for 30 minutes in the dark with monoclonal antibodies against human kinase insert domain receptor (KDR) which was phycoerythrin (PE)-labeled (10 $\mu$; R\&D Systems, Minneapolis, MN), and for 10 minutes with CD34 (fluorescein isothiocyanate [FITC]-labeled)
(20 $\mu 1$; Becton Dickinson), and CD133 (APC-labeled) ( $20 \mu \mathrm{l}$; Miltenyi, Auburn, CA). EPC were defined as cells positive for monoclonal antibodies against human KDR, CD34, and CD133. ${ }^{1-3}$ These bone-marrow-derived hematopoetic progenitor cells differentiate into mature endothelial cells and contribute to endothelial repair and new vessel formation after ischemic injury. ${ }^{3-6}$ Isotype-identical antibodies IgG1-PE-FITC (Becton Dickinson) and IgG2b-APC (eBioscience, San Diego, CA) served as negative controls. Cell fluorescence was measured immediately after staining. FACSCalibur flow cytometer and CellQuest Software (Becton Dickinson) were used for data acquisition. Data were gated on the lymphocyte population, and 20,000 events were collected in the gated region for each sample. ${ }^{1}$ The percent of $\mathrm{KDR}+/ \mathrm{CD} 34+/ \mathrm{CD} 133+$ cells was expressed as the percent of the gated events.

\section{Flow cytometry for circulating endothelial apoptotic microparticles}

Blood was collected in citrate tubes and processed within one hour of collection. Plasma derived from $10 \mathrm{~mL}$ of blood was centrifuged at $1000 \mathrm{~g}$ for six minutes to generate platelet-poor plasma. $50 \mu \mathrm{L}$ of plasma was incubated with $4 \mu \mathrm{L}$ of PE-labeled monoclonal antibody against CD31 (Becton Dickinson) and $4 \mu \mathrm{L}$ of FITC-labeled CD42b (Becton Dickinson), then diluted with $1 \mathrm{~mL}$ of PBS. PE-labeled IgG1 and FITC-labeled IgG 2a (Pharmingen) served as a negative control. Particle detection was set to trigger by a fluorescent signal greater than background threshold. Data were gated using sizing of microparticles (forward light scatter) in the presence of calibrator beads. EMP were defined as particles $\leq 1.5 \mu \mathrm{m}$ in size positive for monoclonal antibodies against CD31 and negative for CD42b. ${ }^{7}$ FACSCalibur flow cytometer (BD Biosciences) and CellQuest Software were used for data acquisition. Population of CD31+/CD42bmicroparticles smaller than $1.5 \mu \mathrm{m}$ is expressed as the number of EMP per $\mu \mathrm{L}$ of platelet poor plasma. Possible contamination with leukocytes microparticles (CD31+/ CD45+) was assessed. CD31+/CD45+ particles accounted for a negligible percentage of all CD31+ microparticles in samples from both patients with OSA and controls.

\section{Brachial artery flow-mediated dilation}

Vascular response in the brachial artery was assessed by FMD according to the guidelines of the International Brachial Artery Reactivity Task Force. ${ }^{8}$ Brachial artery diameter was measured in the contralateral arm to the endothelial harvesting site. Subjects were evaluated in a 
A
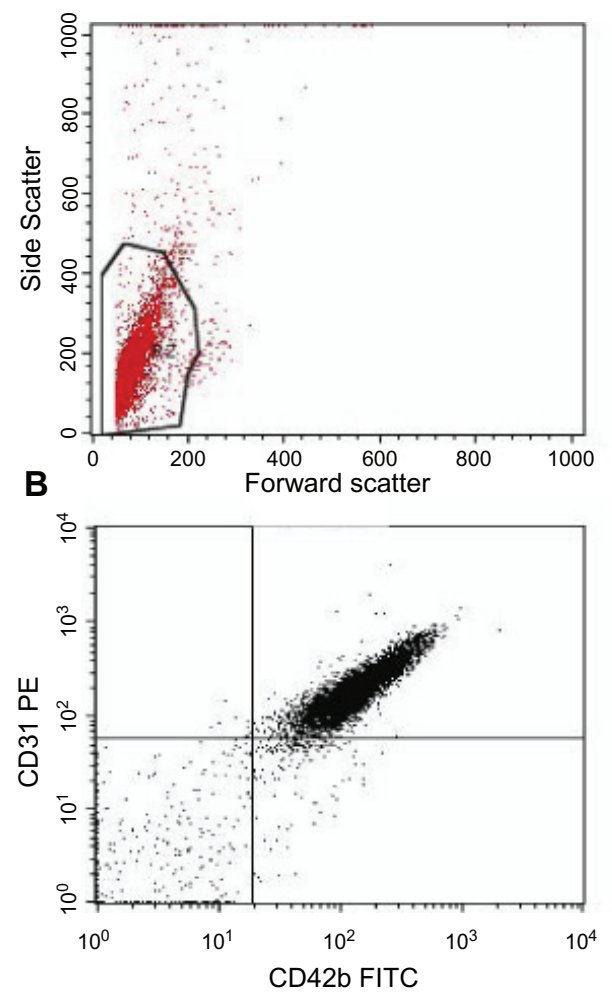

C

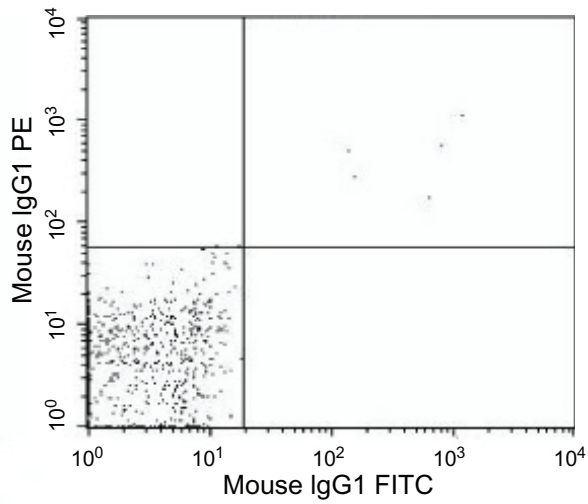

EMP count $=21 /$ microliter
A

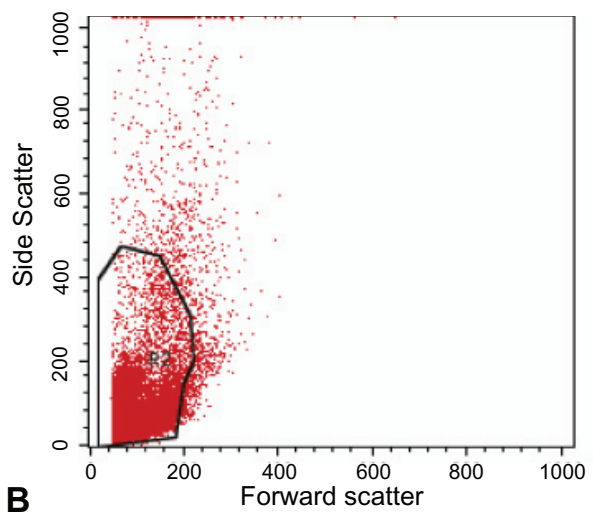

B

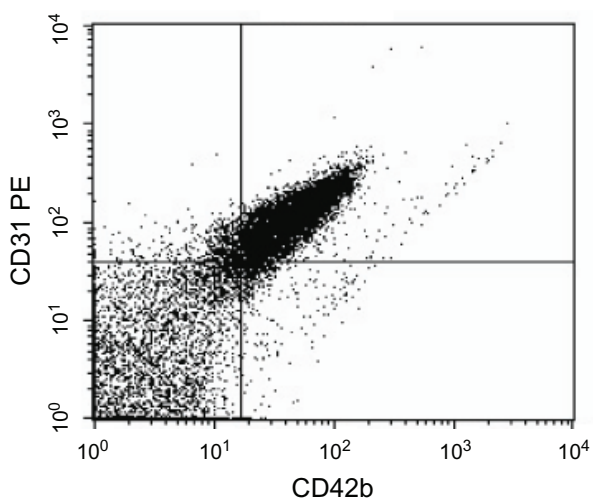

C

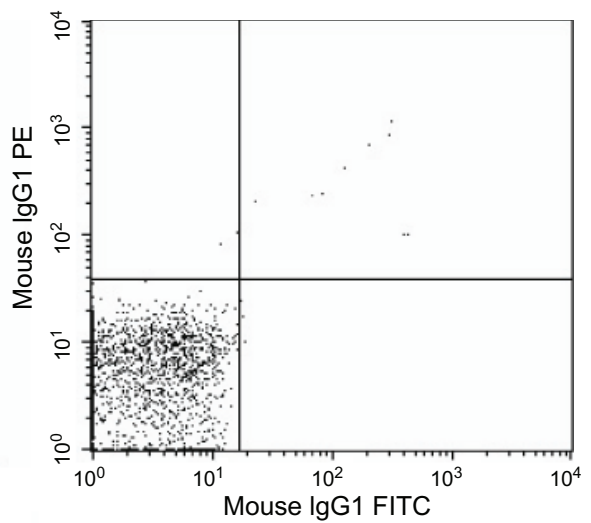

EMP count $=134 / \mathrm{microliter}$

Figure SI Representative histograms of the flow cytometry analysis for endothelial apoptotic microparticles (EMP) from a control subject (Figure IA) and a patient with OSA (Figure IB). Particles were gated based on their size (forward light scatter) in the presence of calibrator beads [region 2 (R2)] A). EMP were defined as particles $\leq$ I.5 $\mu \mathrm{m}$ in size positive for monoclonal antibodies against CD3I and negative for CD42b B). Isotype-identical antibodies served as negative controls C). 
A

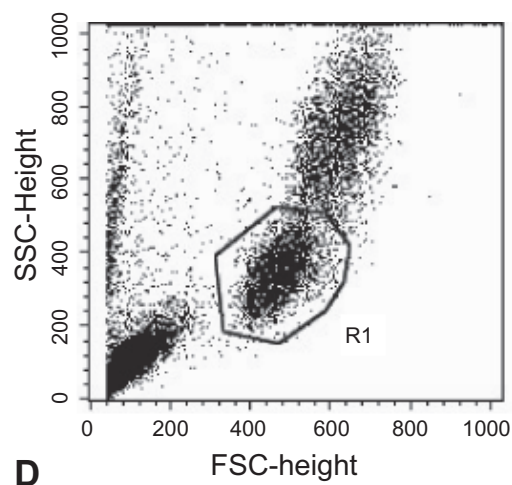

Gated events: 20000 KDR+/CD34+/CD133+cells: 0.10
B
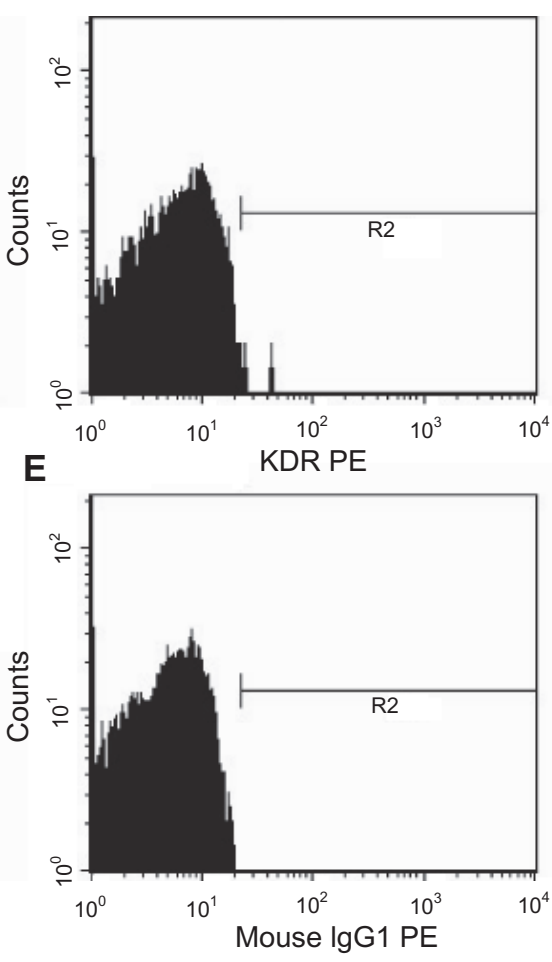

C
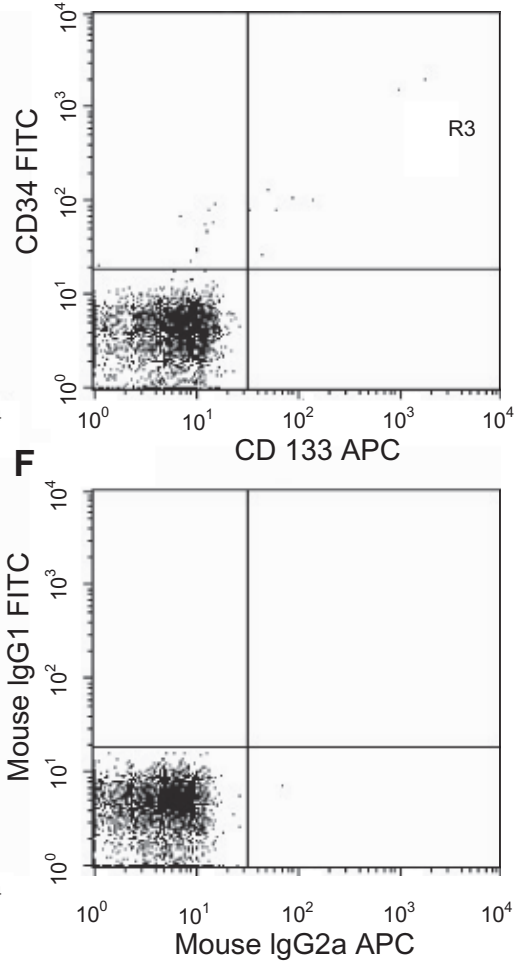

B

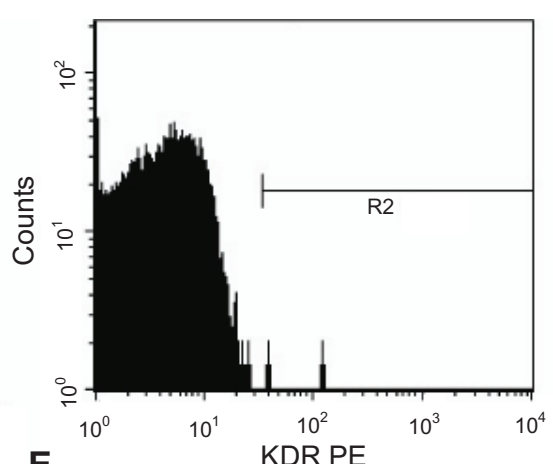

E

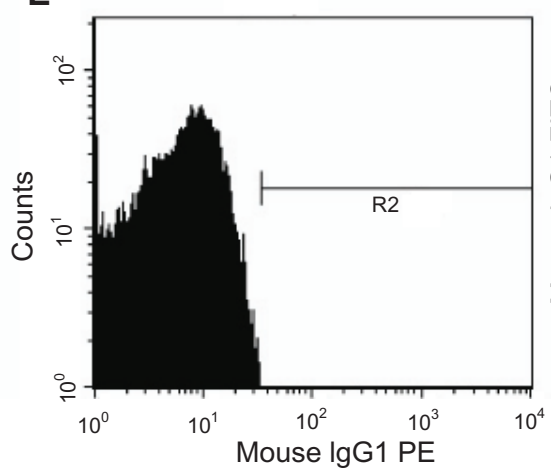

C

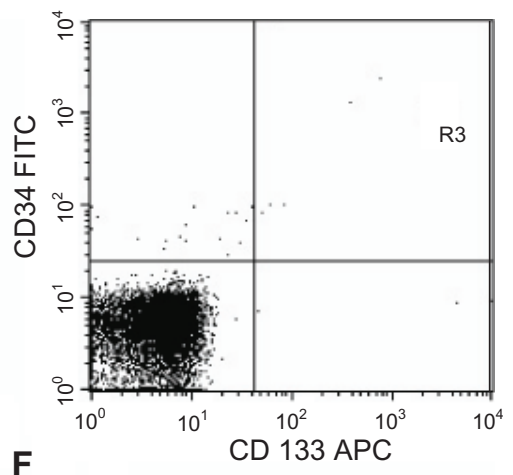

$\mathbf{F}$

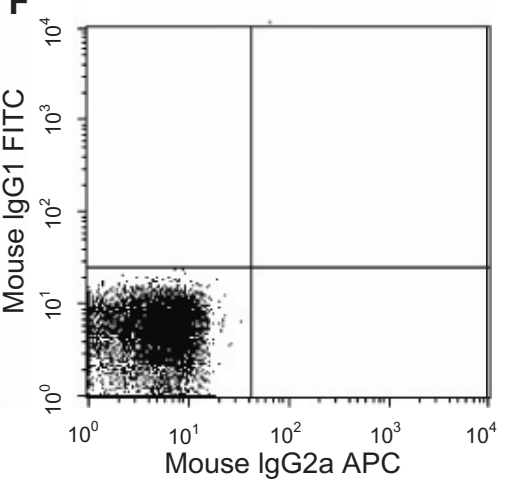

Figure S2 Representative histograms of the flow cytometry analysis for endothelial progenitor cells (EPC) from a control subject (Figure 2A) and a patient with OSA (Figure 2B). Mononuclear lymphocytic cell population was first gated from a plot of forward vs side scatter width [region I(RI)]. A) Those cells were then gated for positive staining for kinase insert domain receptor (KDR) (R2). B) Cells that stained positive for KDR were than gated for double positive staining for CD34 and CDI33 (R3). C) The percent of KDR+/CD34+/CD I33+ cells was expressed as the percent of the gated events, D) Isotype-identical antibodies IgG I-PE, E) and IgG I-FITC and IgG2b-APC F) served as negative controls. Reproducibility of the measurements was assessed by obtaining two separate blood samples from eight control subjects on days 0 and 28 . The overall coefficient of variation between the two measurements was $10 \%$. 
quiet, temperature-controlled room. After a 30-minute rest in a supine position, the brachial artery diameter was measured $6 \mathrm{~cm}$ proximal to the antecubital fossa using a 7-15 MHz linear array transducer (Philips 5500, Andover, MA). Occlusion blood pressure cuff was placed over the proximal forearm just below the antecubital fossa. FMD was measured as the dilator response to reactive hyperemia induced by a five-minute blood pressure cuff occlusion of the upper arm. The cuff was inflated to $50 \mathrm{~mm} \mathrm{Hg}$ above systolic blood pressure if the systolic blood pressure was greater than $150 \mathrm{~mm} \mathrm{Hg}$, or to $200 \mathrm{~mm} \mathrm{Hg}$ if the systolic blood pressure was less than $150 \mathrm{~mm} \mathrm{Hg}$. Systolic blood pressure was lower than $150 \mathrm{~mm} \mathrm{Hg}$ in all study participants. Brachial artery diameter (expressed in millimeters up to one decimal place) was measured at rest and during peak hyperemia for one minute after a five-minute occlusion of arterial flow. A blinded reader analyzed brachial artery diameters off-line using analysis software. The percent diameter change for FMD was calculated as follows:

FMD $(\%)=[($ brachial artery diameter at peak hyperemia - diameter at rest)/diameter at rest] $\times 100$.

\section{References}

1. Werner N, Kosiol S, Schiegl T, et al. Circulating endothelial progenitor cells and cardiovascular outcomes. $N$ Engl J Med. 2005;353: 999-1007.

2. Urbich C, Dimmeler S. Endothelial progenitor cells: characterization and role in vascular biology. Circ Res. 2004;95:343-353.

3. Peichev M, Naiyer AJ, Pereira D, et al. Expression of VEGFR-2 and AC133 by circulating human CD34(+) cells identifies a population of functional endothelial precursors. Blood. 2000;95:952-958.

4. Asahara T, Murohara T, Sullivan A, et al. Isolation of putative progenitor endothelial cells for angiogenesis. Science. 1997;275:964-967.

5. Schachinger V, Erbs S, Elsasser A, et al; REPAIR-AMI Investigators. Intracoronary bone marrow-derived progenitor cells in acute myocardial infarction. N Engl Med. 2006;355:1210-1221.

6. Erbs S, Linke A, Schachinger V, et al. Restoration of microvascular function in the infarct-related artery by intracoronary transplantation of bone marrow progenitor cells in patients with acute myocardial infarction: the Doppler Substudy of the Reinfusion of Enriched Progenitor Cells and Infarct Remodeling in Acute Myocardial Infarction (REPAIR-AMI) trial. Circulation. 2007;116:366-374.

7. Ferreira AC, Peter AA, Mendez AJ, et al. Postprandial hypertriglyceridemia increases circulating levels of endothelial cell microparticles. Circulation. 2004;110:3599-3603.

8. Corretti MC, Anderson TJ, Benjamin EJ, et al; International Brachial Artery Reactivity Task Force. Guidelines for the ultrasound assessment of endothelial-dependent flow-mediated vasodilation of the brachial artery: a report of the International Brachial Artery Reactivity Task Force. J Am Coll Cardiol. 2002;39:257-265.
Vascular Health and Risk Management

\section{Publish your work in this journal}

Vascular Health and Risk Management is an international, peerreviewed journal of therapeutics and risk management, focusing on concise rapid reporting of clinical studies on the processes involved in the maintenance of vascular health; the monitoring, prevention and treatment of vascular disease and its sequelae; and the involvement of

\section{Dovepress}

metabolic disorders, particularly diabetes. This journal is indexed on PubMed Central and MedLine. The manuscript management system is completely online and includes a very quick and fair peer-review system, which is all easy to use. Visit http://www.dovepress.com/ testimonials.php to read real quotes from published authors. 